\title{
MagicHand: In-Hand Perception of Object Characteristics for Dexterous Manipulation
}

\author{
Hui Li,* Hongsheng He \\ ${ }^{1}$ Department of Electrical Engineering and Computer Sciences, College of Engineering
}

Perception of object materials improves the understanding of object characteristics such as fragility, rigidity, texture, mass and density, therefore facilitating and innovating robotic grasping. In this work, a novel way is proposed to identify these important characteristics for dexterous grasping. Near-infrared (NIR) spectra were collected for six types of materials including ceramic, stainless steel, wood, cardboard, plastic and glass using the in-hand SCiO sensor. A deep-learning model is designed to classify the spectra. The proposed approach is evaluated for dexterous grasping based on the perceived object characteristics. The experiment results achieve $99.96 \%$ accuracy on material recognition. In the grasping experiment, the robotic hand selected different grasping strategies for objects that are with similar dimensions but made of different materials. The results showed improved grasping strategies based on characteristics of objects perceived by our algorithm.

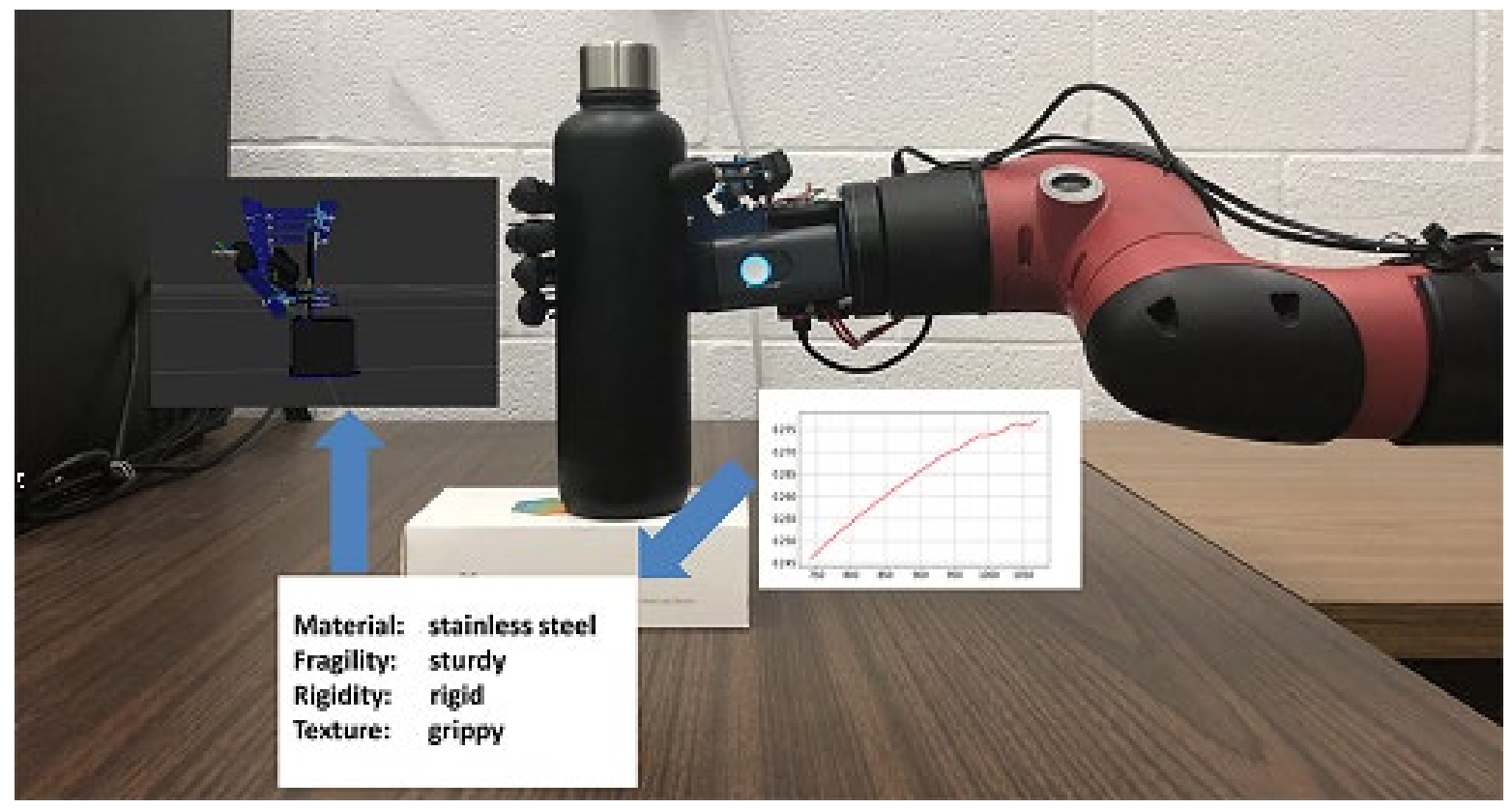

